УДК 544.7

\title{
МОДИФИЦИРОВАННЫЕ ПОЛИЭТИЛЕНПОЛИАМИНОМ И ЭПОКСИДНОЙ СМОЛОЙ ЦЕОЛИТЫ ДЛЯ ИЗВЛЕЧЕНИЯ ИОНОВ СВИНЦА ИЗ СТОЧНЫХ ВОД
}

\author{
Камбарова Эльмира Абдувалиевна ${ }^{1}$, \\ ilmira080884@mail.ru
}

\section{Гавриленко Михаил Алексеевич2,} dce@mail.ru

\author{
Бектенов Несипхан Абжапарович³, \\ bekten_1954@mail.ru \\ 1 Таразский Государственный университет им. М.Х. Дуалти, \\ Казахстан, 080000, г. Тараз, ул. Толе би, 60. \\ 2 Национальный исследовательский Томский политехнический университет, \\ Россия, 634050, г. Томск, пр. Ленина 30. \\ 3 Казахский национальный педагогический университет имени Абая, \\ Казахстан, 050000, г. Алматы, пр. Достык, 13.
}

\begin{abstract}
Актуальность. Создание эфффективных и недорогих сорбентов широкого профриля, одним из актуальных направлений которого является дизайн сорбентов для очистки вод различного назначения, постоянно востребовано в науке и производстве. Новые ионообменные сорбенты позволяют решать сразу две связанные задачи: очистка технической воды предприятий и регулирование содержания токсикантов в окружающей среде. Модифицирование ионообменников приводит к улучшению сорбционных и кинетических характеристик, особенно перспективны природные высокопроницаемые сетчатье полиэлектролиты с большой скоростью поглощения ионов крупных размеров. Модисицирование этих минералов поверхностным слоем сополимеров с эпоксидными группами, полученными при отверждении смеси полиэтиленполиамина и эпоксидной смолы, позволяет получить новые дешевые сорбционные материалы для очистки воды от тяжелых металлов, преимущественно катионов свинца $\mathrm{Pb}^{2+}$. Исследование обусловлено необходимостью очистки сточных вод металлургических предприятий от катионов $\mathrm{Pb}^{2+}$, которые относятся к токсичным веществам, проявляющим мутагенные, канцерогенные свойства, кроме того, встроенные в биогенные формы ионы обладают свойством локально накапливаться в природных объектах, тем самым являясь сильным экотоксикантом.

Цель: определить сорбционные свойства природного цеолита и его модифицированной фрормы по отношению к иону $\mathrm{Pb}^{2+}$ u оптимизировать условия сорбции.

Объекты: Шанханайский природньй цеолит и его модифрицированные полиэтиленполиамином (ПЭПА) и эпоксидной смолой (ЭД-20) формы. Методы: спектрофотометрия.

Результаты. Получены модифицированные полиэтиленполиамином и эпоксидной смолой сорбенты на основе Шанханайского природного цеолита. Установлены оптимальные условия $\mathrm{pH}$ сорбции ионов $\mathrm{Pb}^{2+}$ в статических условиях. При использовании модифицированного природного цеолита в диапазоне рН 7-9 извлечение ионов свинца происходит на $90 \%$ по сравнению с исходным цеолитом. Модифицированный природньй цеолит может быть использован в качестве сорбционного материала для очистки промышленных и сточных вод.
\end{abstract}

\section{Ключевые слова:}

Шанханайский природный цеолит, сорбция, катион $\mathrm{Pb}^{2+}$, полиэтиленполиамин, эпоксидная смола.

\section{Введение}

Ионы свинца являются стойкими экотоксикантами с низкой скоростью удаления из экосистем и организма человека. Значительная биоаккумуляция привела к включению ионов свинца $\mathrm{Pb}^{2+}$ в контрольные списки Евросоюза согласно директиве ЕС 2013/39 $[1,2]$ по регламенту контроля на содержание ионов тяжелых металлов в объектах окружающей среды. Концентрация $\mathrm{Pb}^{2+}$ в водах рек может колебаться от 0,1 до 100 мкг/л, причем среднее значение обычно не превышает 10 мкг/л. В непроточных водоемах это значение ниже и составляет 4,5 мкг/л [3]. Допустимое содержание $\mathrm{Pb}^{2+}$ в питьевой воде находится в диапазоне 1-60 мкг/л в зависимости от региона, причем в Европе и России не превышает 20 мкг/л [4].

С учетом объемов потребляемой воды, физикохимическая адсорбция является наиболее привлекательной методикой очистки, что обусловлено широ- ким ассортиментом разработанных сорбентов, низким энергопотреблением и высокой эффективностью $[5,6]$. Обычные очистные сооружения эффективны в отношении органических веществ, однако удаление ионов тяжелых металлов не всегда происходит полностью, что делает необходимыми доочистку и контроль качества воды. Синтетические ионообменные материалы являются эффективными сорбентами для $\mathrm{Pb}^{2+}$ и других тяжелых металлов, причем наиболее распространенными становятся модифицированные ионообменники [7]. Разработанные и распространенные коммерческие сорбенты обеспечивают высокие скорости удаления, но их высокая стоимость является недостатком в крупнотоннажных системах. Таким образом, поиск альтернативы в виде недорогих природных сорбентов с минимальным модифицированием поверхности доступными реагентами является насущной необходимостью [8]. Исследования проде- 
монстрировали эффективность использования для этой цели активированного угля, углеродных нанотрубок, природных и синтетических цеолитов [9, 10]. Разработка казахстанских природных месторождений цеолитов предоставляет новые природные материалы как для интенсивного развития фундаментальных исследований сорбентов, так и для практического применения. Исследование химического и фазового состава цеолитовых туфов Тайжургенского и Шанханайского месторождений показало возможность практического использования казахстанских цеолитов [11]. Установлено, что такие цеолиты с высоким содержанием кремнезема являются эффективными адсорбентами для очистки загрязненной воды 「12]. Например, для удаления ионов тяжелых металлов из воды рекомендован мезапористый цеолитный сорбент фожазит, поверхность которого обработана щелочью [13].

Другой цеолит, модифицированный смесью окисленных полиэтилена и полипропилена, использовали для удаления ионов $\mathrm{Pb}^{2+}$ и $\mathrm{Cu}^{2+}$ из воды [14]. Исследован процесс очистки воды от $\mathrm{Ni}^{2+}, \mathrm{Co}^{2+}, \mathrm{Pb}^{2+}$ и $\mathrm{Cu}^{2+}$ природным цеолитом Ягоднинского месторождения Камчатской области в диапазоне концентраций в воде 0,5-3,5 мг-экв/л. Установлено, что модифицирование поверхности серной кислотой приводит к активации функциональных групп и увеличению его эффективности [15]. Такие результаты свидетельствуют о том, что модифицированные природные цеолиты являются перспективными недорогими материалами для очистки воды от тяжелых металлов [16].

Аналитические методы определения ионов $\mathrm{Pb}^{2+}$ включают в себя использование пламенной и электротермической атомно-абсорбционной спектрометрии, атомно-абсорбционной спектрометрии с графитовой печью (GFAAS), атомной индуктивно-связанной плазмой эмиссионной спектрометрии (ICP-AES) и спектрометрии с индуктивно-связанной плазмой (ICP-MS) [17-21]. Эти методы имеют достаточную чувствительность, однако некоторые из них очень дороги и недоступны для обычной аналитической лаборатории. Низкие концентрации катионов свинца могут быть обнаружены методом флуоресцентной спектрометрии [22] с высокой степенью точности и повторяемости, в короткое время анализа [23]. Для большинства методов низкий уровень токсичного присутствия металла не соответствует инструментальной чувствительности, что требует использования предварительного концентрирования на сорбентах. Наиболее эффективным по соотношению чувствительности аналитических характеристик и трудоемкости использования является спектрофотометрический метод [24-27]. Увеличить чувствительность данного вида анализа можно за счет добавления селективного органического реагента, который переводит определяемый ион в хромогенное соединение [28].

Объектом настоящего исследования является оценка изменения сорбционных свойств Шанханайского природного цеолита и его модифицированных полиэтиленполиамином (ПЭПА) и эпоксидной смолой (ЭД-20) форм по отношению к $\mathrm{Pb}^{2+}$ из-за таких факторов, как $\mathrm{pH}$ среда, концентрация иона в растворе и время контакта.

\section{Экспериментальная часть}

Модифицирование цеолита

Шанханайский цеолит получен из месторождения Республики Казахстан, Алматинская область, Кербулакский район, г. Сары-Озек. Перед модификацией предварительно минерал измельчали до диаметра зерен 0,4 мм. Для повышения извлекающей способности и селективности природного Шанханайского цеолита сорбент модифицировали полиэтиленполиамином (ПЭПА, Россия, ТУ 2413-357-00203447-9920) и эпоксидной смолой (ЭД-20, Россия, ГОСТ 10587-84). К 100 г полученного цеолитного порошка добавляли ЭД-20 массой 30 г до полного покрытия поверхности, затем небольшими порциями добавляли 30 г ПЭПА и перемешивали в течение 10 мин. Полученную массу выгружали в фарфоровые чашки и отверждали в сушильном шкафу в течение 10 ч при $120{ }^{\circ} \mathrm{C}$. Затем массу вынимали и охлаждали при комнатной температуре в течение 10 ч. Образцы полученного модифицированного цеолита обрабатывали $5 \%$ раствором $\mathrm{HCl}$ для регенерации хлоридной формы, затем путем отмывки водой доводили до нейтральной $\mathrm{pH}$ и обрабатывали $5 \%$ раствором $\mathrm{NaOH}$. Полученный модифицированный цеолит сушили до постоянного веса в муфельной печи при $120{ }^{\circ} \mathrm{C}$. Анионообменную емкость продукта (COE, мг-экв/Г) определяли в статических условиях по 0,1 Н раствору НСI [29].

Реактивы, растворы, материалы

Спектрофотометрическое определение $\mathrm{Pb}^{2+}$ в растворе после сорбции связано с образованием комплексного соединения, которое можно охарактеризовать взаимодействием ионов $\mathrm{Pb}^{2+}$ с сульфарсазеном. Данное комплексное соединение окрашено в желтооранжевый цвет [30]. В работе использованы растворы 10 мг/л $\mathrm{Pb}\left(\mathrm{NO}_{3}\right)_{2}, 0,05 \mathrm{M} \mathrm{Na}_{2} \mathrm{~B}_{4} \mathrm{O}_{7}, 0,1 \mathrm{MHNO}_{3}$, $0,1 \mathrm{M} \mathrm{NaOH}$. Растворы исходных солей готовили по точной навеске, растворы меньших концентраций последовательным разбавлением исходных растворов непосредственно перед использованием. Растворы 0,05\% сульфарсазена готовили растворением в 0,05 моль/л $\mathrm{Na}_{2} \mathrm{~B}_{4} \mathrm{O}_{7}$. Для всех растворов использована бидистилированная вода.

Статическую сорбционную емкость сорбента (А) рассчитывали по формуле:

$$
A\left(\mathrm{~Pb}^{2+}\right)=\frac{\left(C_{\text {исх }}-C_{\text {равн }}\right)}{m} \cdot V,
$$

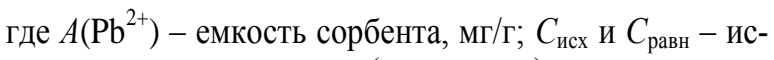
ходная и равновесная (остаточная) концентрации ионов металлов в растворе соответственно, мг/л; $V-$ объем раствора, л; $m$ - масса сорбента, г.

Степень извлечения ионов металлов $(E, \%)$ рассчитывали по формуле:

$$
E=\frac{\left(C_{\text {исх }}-C_{\text {равн }}\right)}{C_{0}} 100 \%,
$$


где $C_{\text {исх }}$ - исходная концентрация иона металла в растворе, мг/л; $C_{\text {равн }}-$ равновесная (остаточная) концентрация иона металла в растворе, мг/л.

\section{Аппаратура и приборы}

Спектрофотометрические измерения проводили на спектрофотометре UV-1800 (Shimadzu, Япония). Оптическую плотность измеряли при длине волны 530 нм в кюветах толщиной слоя 1 см. Для перемешивания использовали мультиротатор MultiBio RS-24 (BioSan, Латвия).

\section{Результаты и их обсуждение}

Изучена сорбция ионов $\mathrm{Pb}^{2+}$ цеолитом и его модифицированными ПЭПА и ЭД-20 формами в интервале $\mathrm{pH}$ от 1,68 до 12,45. Количественное определение ионов свинца в водных растворах проводили по предварительно построенному градуированному графику (рис. 1). Исследование показало, что максимальная сорбционная емкость (рис. 2) модифицированной формы (МФ) в данных условиях по $\mathrm{Pb}^{2+}$ составляет $0,5 \mathrm{M \Gamma} / \Gamma$, для природного цеолита (ПЦ) 0,25 мг/г при рН 6,86, в этих условиях сорбция МФ выше, чем на ПЦ, на 50 \%. При рН 9,18 сорбция на МФ выше на 90 \%. Изучение влияния $\mathrm{pH}$ на величину сорбции ионов $\mathrm{Pb}^{2+}$ из водных растворов на ПЦ и его МФ в зависимости от рН среды показало, что оптимальной кислотностью среды для сорбции свинца является интервал рН 6,86-9,18. Выше этого интервала наблюдают образование гидроксильных комплексов ионов свинца, ниже интервала использование сульфарсазена приводит к появлению комплексов нестабильного состава.

\section{A}

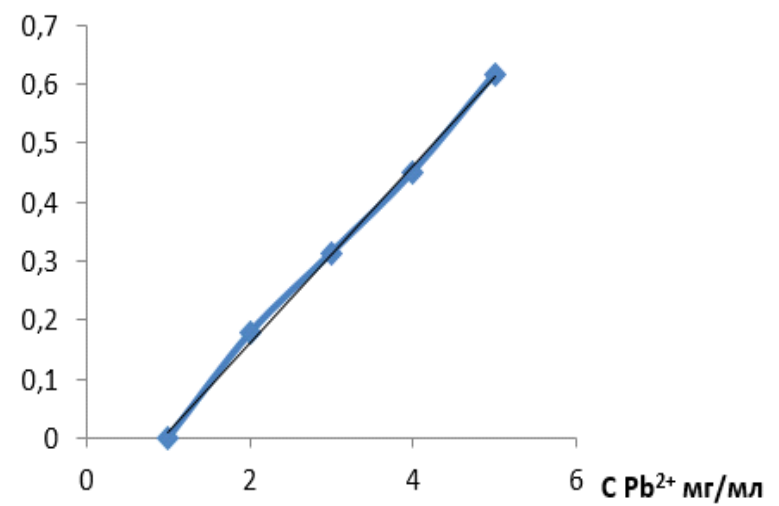

Рис. 1. Градуировочная зависимость для определения $\mathrm{Pb}^{2+}$ сульфарсазеном в водном растворе

Fig. 1. Calibration dependence for $\mathrm{Pb}^{2+}$ determination by sulfarsazene in aqueous solution

Продолжительность контакта сорбента с раствором $\mathrm{Pb}^{2+}$ влияет на степень извлечения (рис. 3). С увеличением длительности контакта величина сорбции повышается, затем после 90 мин становится постоянной.

По результатам модифицирования природного цеолита с ПЭПА и ЭД-20 доказано повышение сорбционной емкости к ионам $\mathrm{Pb}^{2+}$, что проявляется в изменении изотермы сорбции (рис. 4).

\section{$\mathrm{C} \mathrm{Pb2+,Mr/r}$}

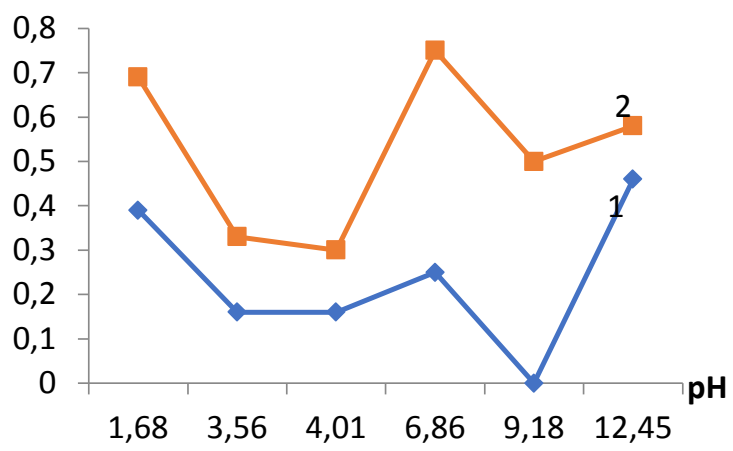

Pис. 2. Сравнительная характеристика сорбции $\mathrm{Pb}^{2+}$ природного иеолита (1) и его модифицированной формы (2) в зависимости от рН среды

Fig. 2. Comparative characteristics of $\mathrm{Pb}^{2+}$ sorption of natural zeolite (1) and its modified form (2) depending on medium $\mathrm{pH}$

\section{$\mathrm{CPb} 2+, \mathrm{Mr} / \mathrm{r}$}

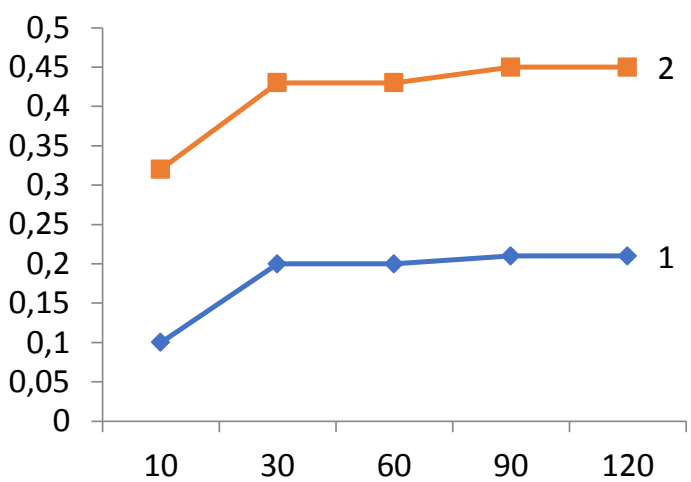

t, мин

Pис. 3. Сравнительная характеристика сорбичи $\mathrm{Pb}^{2+}$ природного чеолита (1) и его модифищированной формы (2) при нейтральной среде в зависимости от времени контакта

Fig. 3. Comparative characteristics of $\mathrm{Pb}^{2+}$ sorption of natural zeolite (1) and its modified form (2) in a neutral medium depending on the contact time

Сорбционная ёмкость для $\mathrm{Pb}^{2+}$ значительно выше по сравнению с ПЦ. Количество сорбированного $\mathrm{Pb}^{2+}$ повышается с ростом концентрации исходного раствора. При концентрации $\mathrm{Pb}^{2+}$ в исходном растворе менее 0,01 моль $\cdot$ Л $^{-1}$ достигнута практически полная сорбция $\mathrm{Pb}^{2+}$. Изотерма сорбции ПЦ имеет L-образную форму. Для изотерм класса L характерны равномерное заполнение поверхности и отсутствие конкуренции со стороны растворителя. Также возможен процесс переориентации ассоциатов комплексов свинца относительно поверхности сорбента.

Изотерма сорбции МФ имеет S-образную форму с вогнутым начальным участком. При возрастании концентрации сорбата в растворе увеличивается сорбционная ёмкость сорбента, что связано с изменением ориентации адсорбированных ионов $\mathrm{Pb}^{2+}$ относительно поверхности сорбента или с быстрым переходом к полимолекулярной адсорбции. Далее следует 
точка перегиба и появляется второе плато, что придает таким изотермам характерный S-образный вид. Причина заключается в сильном взаимодействии между адсорбированными катионами $\mathrm{Pb}^{2+}$ при одновременном ослаблении их взаимодействия с поверх-

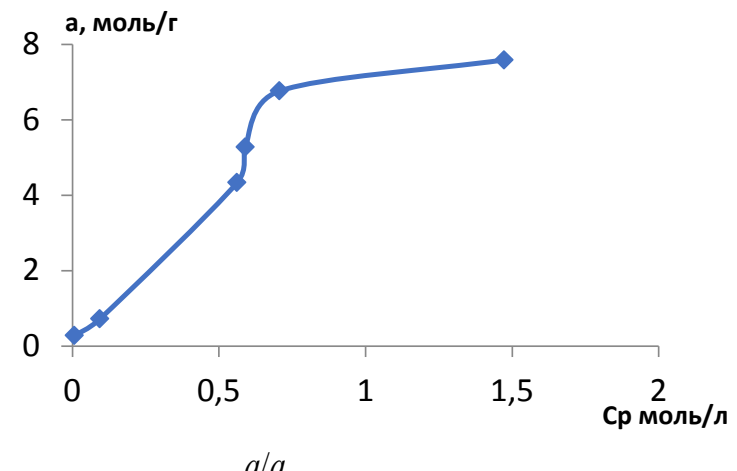

ностью сорбента, в этом случае адсорбированные катионы $\mathrm{Pb}^{2+}$ стремятся расположиться на поверхности в виде скоплений. Экспериментальные данные и параметры изотерм Ленгмюра для сорбции $\mathrm{Pb}^{2+}$ с образцами ПЦ и МФ представлены в таблице.

Рис. 4. Изотерма природного иеолита (а) и его модифииированной формы (б)

Fig. 4. Isotherm of natural zeolite ( $a$ ) and its modified form (b)
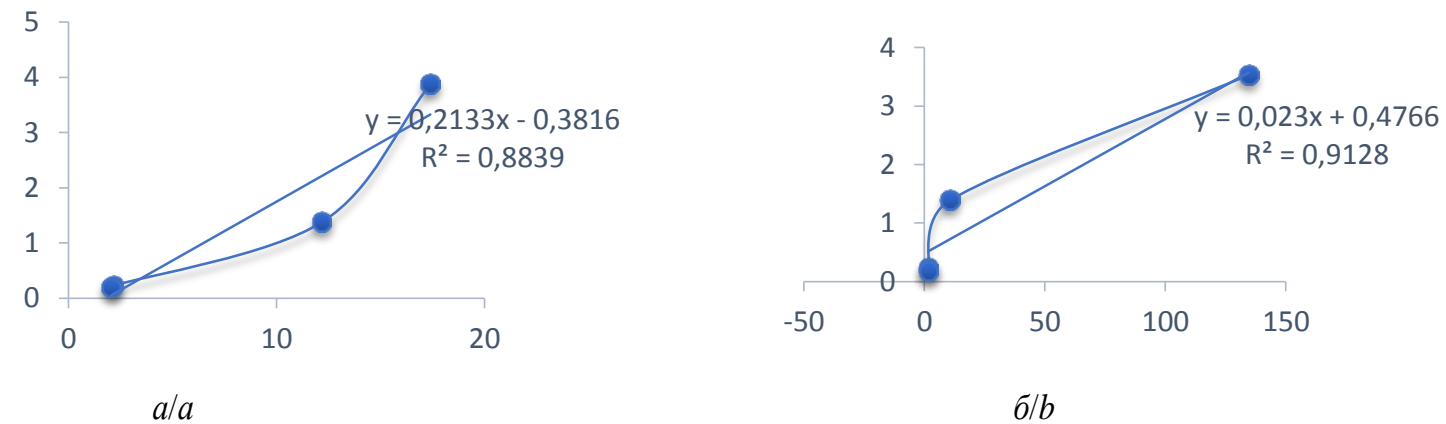

Рис. 5. Параметры изотерм Ленгмюра для сорбиии $\mathrm{Pb}^{2+}$ с образиами модифицированной формы (а) и природного иеолита (б)

Fig. 5. Parameters of the Langmuir isotherms for $\mathrm{Pb}^{2+}$ sorption with samples of modified form (a) and nuture zeolite (b)

таблица. Экспериментальные данные сорбиии $\mathrm{Pb}^{2+}$ с образиами природного цеолита и его модифици рованной формы и результаты их обработки

Table. Experimental data on $\mathrm{Pb}^{2+}$ sorption with natural zeolite and its modified form samples and the results of their processing

\begin{tabular}{|c|c|c|c|c|}
\hline \multicolumn{3}{|c|}{ Модифицированная форма/Modified form } & \multirow{2}{*}{\multicolumn{2}{|c|}{$\begin{array}{l}\text { Уравнение Ленгмюра } \\
\text { The Langmuir equation }\end{array}$}} \\
\hline \multirow{2}{*}{$\begin{array}{c}\mathrm{C}, \text { моль/л } \\
\mathrm{C}, \mathrm{mol} / \mathrm{l}\end{array}$} & \multirow{2}{*}{$\begin{array}{c}\mathrm{C}_{\mathrm{s}}, \text { моль/Л } \\
\mathrm{C}_{\mathrm{s}}, \mathrm{mol} / \mathrm{l}\end{array}$} & \multirow{2}{*}{$\begin{array}{c}\mathrm{a}, \mathrm{моль} / \Gamma \\
\mathrm{a}, \mathrm{mol} / \mathrm{g}\end{array}$} & & \\
\hline & & & $1 / C_{s}$ & $1 / \mathrm{a}$ \\
\hline 0,05 & 0,06 & 0,26 & 17,41 & 3,88 \\
\hline 0,10 & 0,08 & 0,72 & 12,19 & 1,38 \\
\hline 0,25 & 0,46 & 4,38 & 2,18 & 0,23 \\
\hline 0,50 & 0,49 & 5,32 & 2,04 & 0,19 \\
\hline 0,75 & 0,50 & 6,87 & 2,01 & 0,15 \\
\hline 1,00 & 0,22 & 8,21 & 4,45 & 0,12 \\
\hline \multicolumn{3}{|c|}{ Природный цеолит/Natural zeolite } & \multirow{2}{*}{\multicolumn{2}{|c|}{$\begin{array}{l}\text { Уравнение Ленгмюра } \\
\text { The Langmuir equation }\end{array}$}} \\
\hline \multirow{2}{*}{$\begin{array}{c}\mathrm{C}, \text { моль/л } \\
\mathrm{C}, \mathrm{mol} / \mathrm{l} \\
\end{array}$} & \multirow{2}{*}{ 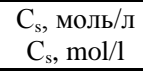 } & \multirow{2}{*}{$\begin{array}{c}\mathrm{a}, \text { моль/г } \\
\mathrm{a}, \mathrm{mol} / \mathrm{l}\end{array}$} & & \\
\hline & & & $1 / C_{s}$ & $1 / \mathrm{a}$ \\
\hline 0,05 & 0,01 & 0,28 & 134,6 & 3,54 \\
\hline 0,10 & 0,09 & 0,72 & 10,67 & 1,40 \\
\hline 0,25 & 0,56 & 4,33 & 1,78 & 0,23 \\
\hline 0,5 & 0,59 & 5,27 & 1,70 & 0,19 \\
\hline 0,75 & 0,71 & 6,76 & 1,41 & 0,15 \\
\hline 1,00 & 1,47 & 7,58 & 0,68 & 0,13 \\
\hline
\end{tabular}

Графические и расчетные данные показывают возрастание сорбционной емкости модифицированного цеолита, что связано с увеличением количества функциональных групп на поверхности сорбента.

\section{Заключение}

Получен сорбент на основе Шанханайского природного цеолита и его модифицированных полиэтиленполиамином (ПЭПА) и эпоксидной смолой (ЭД-20) форм. Установлена оптимальная $\mathrm{pH}$ от 7 до 9 для очистки воды от ионов свинца. В указанном диапазоне $\mathrm{pH}$ при использовании модифицированного природного цеолита происходит извлечение ионов свинца на 90 \%. Таким образом, для достижения полноты извлечения необходимо в два раз меньше модифицированного сорбента в нейтральной среде и в пять раз меньше в слабощелочной среде. Время сорбции не превышает 90 мин. Модифицированный природный цеолит может быть эффективно использован в качестве сорбционного материала для очистки сточных вод. 


\section{СПИСОК ЛИТЕРАТУРЫ}

1. Tuzen M., Soylak M., Parlar K. Cadmium and lead contamination in tap water samples from Tokat, Turkey // Bull. Environ. Contam. Toxicol. - 2005. - V. 75 (2). - P. 284-289.

2. Опасен ли свинец в воде? / И.Н. Андрусишина, И.А. Голуб, 3.В. Малецкий, И.П. Лубянова // Вода и водоочистные технологии. Научно-технические вести. - 2016. - № 2. - С. 40-50.

3. ГОСТ 24902-81. Вода хозяйственно-питьевого назначения Общие требования к полевым методам анализа. - М.: Стандартинформ, 1999. - 114 c.

4. WHO guidelines for drinking water quality. V. 2. 2nd ed. Geneva: World Health Organization, 1996. - 973 p.

5. Liang P., Sang H. Determination of trace lead in biological and water samples with dispersive liquid-liquid microextraction preconcentration // Analytical Biochemistry. - 2008. - V. 380. Р. 21-25.

6. Исследование фазового и химического состава природных цеолитов и катализаторов на их основе / К.А. Жубанов, Р.М. Бабусенко, В.Ф. Тимофеева, К.Н. Солохина // Известия Национальной академии наук Республики Казахстан. Серия химии и технологии. - 2000. - № 5. - С. 158-162.

7. Water purification from heavy metals with ionites / N.A. Bektenov, I.G. Tsoy, E.A. Kambarova, L.K. Ybyrayymzhanova // The scientific heritage. - 2019. - V. 1. - № 38. - P. 30-34.

8. Zaiku X., Qingling C., Chengfang Z. Influence of citric acid treatmenton the surface acid properties of zeolite beta $111 / /$ Physical Chemistry. - 2000. - V. 104 B. - № 13. - P. 2853-2859.

9. Состав и термоустойчивость цеолитсодержащего туфа Шанханайского месторождения / К.А. Жубанов, Р.М. Бабусенко, В.Ф. Тимофеева, Н.Н. Солохина // Вестник Казахского национального университета имени аль-Фараби. Серия химия 2004. - № 3. - С. 75-78.

10. Курбангалиева Г.В., Кудереева Л.А., Конусбаев С.Р. Влияние кислотной активации на физико-химические свойства природных цеолитов // Вестник Казахского национального университета имени аль-Фараби. Серия химия. - 2004. - Т. 34. № 2. - С. 52-56.

11. Ергожин Е.Е., Акимбаева А.М. Органоминеральные сорбенты и полифункциональные системы на основе природного алюмосиликатного и угольно-минерального сырья. - Алматы: Изд-во ТОО «Print-S», 2007. - 373 с.

12. High-silica zeolites for adsorption of organic micro-pollutants in water treatment / Nan Jiang, Ran Shang, S.G.J. Heijman, C. Luuk // A review Water Research. - 2018. - V. 144. - P. 145-161.

13. Sulaiman K.O., Sajid M., Alhooshani K. Application of porous membrane bag enclosed alkaline treated Y-Zeolite for removal of heavy metal ions from water // Microchemical Journal. - 2020. V. 152. - P. 124-135.

14. Molecular dynamics simulation of water purification using zeolite MFI nanosheets / S. Rassoulinejad-Mousavi, J. Azamat, A. Khataee, Y. Zhang // Separation and Purification Technology. 2020. - V. 234. - 116080.

15. Belova T.P. Adsorption of heavy metalions $(\mathrm{Cu} 2+, \mathrm{Ni} 2+, \mathrm{Co} 2+$ and $\mathrm{Fe} 2+$ ) from aqueous solutions by natural zeolite // Heliyon. 2019. - V. 5. - 02320 .

16. Development of HZSM-12 zeolite for catalytic degradation of high-densitypolyethylene / A.O.S. Silva, M.J.B. Souza A.M.G. Pedrosa, A.C.F. Coriolano, A.S. Araujo // Microporous and Mesoporous Materials. - 2017. - V. 244. - P. 1-6.

17. Experimental study of catalytic pyrolysis of polyethylene and polypropylene over USY zeolite and separation to gasoline and diesel-like fuels / C. Kassargy, S. Awad, G. Burnens, K. Kahine //
Journal of Analytical and Applied Pyrolysis. - 2017. - V. 127. P. 31-37.

18. Intensification of supercritical water oxidation ( $\mathrm{ScWO})$ process for landfill leachate treatment through ion exchange with zeolite / A.P. Jambers Scandelai, J.P. Zotesso, V. Jegatheesan, L. CardozoFilho, C. Tavares // Waste Management. - 2020. - V. 101. P. 259-267.

19. Sorption of $\mathrm{Pb}$ (II) from aqueous solutions by acid-modified clinoptilolite-rich tuffs with different $\mathrm{Si} / \mathrm{Al}$ ratios / M. Abatal, A.V. Quiroz Córdova, M.T. Olguín, A.R. Vázquez-Olmos, J. Vargas, F. Anguebes-Franseschi, G. Giácoman-Vallejos // Applied sciences. - 2019. - V. 9. - P. 2415-2432.

20. Manzoori J.L., Amjadi M., Abulhassan J. Ultra-trace determination of lead in water and food samples by using ionic liquid-based single drop microextraction-electrothermal atomic absorption spectrometry // Analytical chemistry. - 2009. Acta 644. - P. 48-52.

21. Simultaneous pre-concentration procedure for the determination of cadmium and lead in drinking water employing sequential multielement flame atomic absorption spectrometry / L.A. Portugal, H.S. Ferreira, W.N.L. Santos dos, S.L.C. Ferreira // Microchemical. - 2007. - J. 87. - P. 77-80.

22. Biasino J., Domínguez J.R., Alvarado J. Hydrogen peroxide in basic media for whole blood sample dissolution for determination of its lead content by electrothermal atomization atomic absorption spectrometry // Talanta. - 2007. - V. 73 (5). - P. 962-964.

23. Comparison between the calibration and the standard addition methods in determining dissolved lead in Borobudur's control tanks water by Flame Atomic Absorption Spectrophotometry (F-AAS) / I. Sulistyaningrum, M.P. Gitutami, R.B. Istiningrum, I.M. Siregar // Procedia Chemistry. - 2015. - V. 17. - P. 70-74.

24. Zhou Q., Zhao N., Xie G. Determination of lead in environmental waters with dispersive liquid-liquid microextraction prior to atomic fluorescence spectrometry // Journal of Hazardous Materials. - 2011. - V. 89 (1-2). - P. 48-53.

25. Automated dispersive liquid-liquid microextraction coupled to high performance liquid chromatography - cold vapour atomic fluorescence spectroscopy for the determination of mercury species in natural water samples / Yao-Min Liu, Feng-Ping Zhang, Bao-Yu Jiao, Jin-Yu Rao, Geng Leng // Journal of Chromatography A. - 2017. - V. 1493. - P. 1-9.

26. Radhakrishnan K., Panneerselvam P., Sivanesan S. Turn-on fluorescence sensor based detection of heavy metal ion using carbon dots graphitic-carbon nitride nanocomposite probe // Journal of Photochemistry and Photobiology A: Chemistry 389. 2019. - $112204 \mathrm{~K}$.

27. Sequential determination of lead and cobalt in tap water and foods samples by fluorescence / M.C. Talio, M. Alesso, M. Acosta, M.G. Acosta, L.P. Fernández // Talanta. - 2014. - V. 127 P. 244-249.

28. Tarighat M.A. Orthogonal projection approach and continuous wavelet transform-feed forward neural networks for simultaneous spectrophotometric determination of some heavy metals in diet samples // Food Chemistry. - 2016. - V. 192. - P. 548-556

29. ГОСТ 20255.1-89. Иониты. Метод определения статической обменной емкости. - М.: Стандартинформ, 2002. - 5 с.

30. Rosenstein C., Hirsch S. Chemical analysis of plating solutions // Metal Finishing. - 2002. - V. 100. - P. 509-554.

Поступила 18.11.2020 г.

\section{Информация об авторах}

Камбарова Э.А., аспирант отделения химической инженерии, Таразский Государственный университет им. М.Х. Дуалти.

Гавриленко М.А., доктор химических наук, профессор отделения химической

инженерии Инженерной школы природных ресурсов Национального исследовательского Томского политехнического университета.

Бектенов Н.А., доктор химических наук, профессор Казахского национального педагогического университета имени Абая. 
UDC 544.7

\title{
ZEOLITES MODIFIED WITH POLYETHYLENE POLYAMINE AND EPOXY RESIN TO EXTRACT LEAD IONS FROM WASTEWATER
}

\author{
Elmira A. Kambarova ${ }^{1}$, \\ ilmira080884@mail.ru
}

\author{
Mikhail A. Gavrilenko², \\ dce@mail.ru
}

\author{
Nesipkhan A. Bektenov3, \\ bekten_1954@mail.ru
}

1 Taraz State University named after M.Kh. Dualty, 60, Tole bi street, Taraz, 080000, Kazakhstan.
2 National Research Tomsk Polytechnic University, 30, Lenin avenue, Tomsk, 634050, Russia.
3 Kazakh National Pedagogical University named after Abay, 13, Dostyk avenue, Almaty, 050000, Kazakhstan.

The relevance. Development of effective and inexpensive sorbents of a wide profile is an urgent problem. In this regard, it is necessary to use new modified ion exchangers applied for wastewater treatment and control over the content of various substances in the environment. In order to increase sorption characteristics, modification is performed. Modification of sorbents with improved sorption and kinetic characteristics has great prospects, such as highly permeable cross-linked polyelectrolytes with a high absorption rate of large ions, valuable physicochemical properties, cheap natural minerals modified with polyethylene polyamine and epoxy resins in order to produce reactive copolymers with epoxy groups, capable of participating in curing reactions in the presence of acid compounds and basics. The research is caused by the need for wastewater treatment of metallurgical enterprises from $\mathrm{Pb}^{2+}$ ions. Lead compounds are toxic substances that exhibit mutagenic, carcinogenic properties; in addition, $\mathrm{Pb}^{2+}$ ions embedded in biogenic forms have the property of locally accumulating in natural objects, thereby being a strong ecotoxicant.

The main aim of the research is to determine the sorption properties of natural zeolite and its modified form in relation to the $\mathrm{Pb}^{2+}$ ion and to optimize the sorption conditions.

Objects: Shanghai natural zeolite and its modified polyethylenepolyamine and epoxy resin form.

Methods: spectrophotometry.

Results. Sorbents modified with polyethylene polyamine and epoxy resin based on Shanghai natural zeolite have been obtained. The optimal conditions for the $\mathrm{pH}$ sorption of $\mathrm{Pb}^{2+}$ ions under static conditions were established. When a modified natural zeolite is used in the pH range of 7-9, lead ions are extracted by $90 \%$ in comparison with the original zeolite. Modified natural zeolite can be used as a sorption material for industrial and waste water treatment.

\section{Keywords:}

Shanghai natural zeolite, sorption, $\mathrm{Pb}^{2+}$ cation, polyethylenepolyamine, epoxy resin.

\section{REFERENCES}

1. Tuzen M., Soylak M., Parlar K. Cadmium and lead contamination in tap water samples from Tokat, Turkey. Bull. Environ. Contam. Toxicol., 2005, vol. 75(2), pp. 284-289.

2. Andrusishina I.N., Golub I.A., Maletskiy Z.V., Lubyanova I.P Opasen li svinets v vode? [Is lead in water dangerous?]. Voda $i$ vodoochistnye tekhnologii. Nauchno-tekhnicheskie vesti, 2016, no. 2 , pp. $40-50$

3. GOST 24902-81. Voda khozyaystvenno-pityevogo naznacheniya. Obshchie trebovaniva $k$ polevym metodam analiza [State Standart 24902-81.Drinking water. General requirements for field analysis methods]. Moscow, Standardinform Publ., 1999. 114 p.

4. WHO guidelines for drinking water quality. Vol. 2. $2^{\text {nd }}$ ed. Geneva, World Health Organization, 1996. 973 p.

5. Liang P., Sang H. Determination of trace lead in biological and water samples with dispersive liquid-liquid microextraction preconcentration. Anal. Biochem, 2008, vol. 380, pp. 21-25.

6. Zhubanov K.A., Babusenko P.M., Timofeyeva V.F., Solokhina K.N. Issledovanie fazovogo i khimicheskogo sostava prirodnykh tseolitov i katalizatorov na ikh osnove [Study of the phase and chemical composition of natural zeolites and catalysts based on them]. Izvestiva Natsionalnoy akademii nauk Respubliki Kazakhstan. Seriya khimii i tekhnologii, 2000, no. 5, pp. 158-162.
7. Bektenov N.A.,Tsoy I.G., Kambarova E.A., Ybyrayymzhanova L.K. Water purification from heavy metals with ionites. The scientific heritage, 2019, vol. 38, pp. 30-34.

8. Zaiku X., Qingling C., Chengfang Z. Influence of citric acid treatment on the surface acid properties of zeolite beta 111 . Physical Chemistry, 2000, vol. 104, no. 13, pp. 2853-2859.

9. Zhubanov K.A., Babusenko P.M., Timofeyeva V.F., Solokhina N.N. Sostav i termoustoychivost tseolitsoderzhashchego tufa Shankhanayskogo mestorozhdeniya [Composition and thermal stability of the zeolite-containing tuff of the Shankhanai deposit]. Vestnik Kazakhskogo natsionalnogo universiteta imeni al-Farabi. Seriya khimiya, 2004, no. 3, pp. 75-78.

10. Kurbangaliyeva G.V., Kudereyeva L.A., Konusbayev S.R. Vliyanie kislotnoy aktivatsii na fiziko-khimicheskie svoystva prirodnykh tseolitov [The effect of acid activation on the physicochemical properties of natural zeolites]. Vestnik Kazakhskogo natsionalnogo universiteta imeni al-Farabi. Seriya khimiya, 2004, vol. 34, pp. 52-56.

11. Yergozhin E.E., Akimbayeva A.M. Organomineralnye sorbenty $i$ polifunktsionalnye sistemy na osnove prirodnogo alyumosilikatnogo i ugolno-mineralnogo syrya [Organomineral sorbents and polyfunctional systems based on natural aluminosilicate and coal-mineral raw materials.]. Almaty, TOO «Print-S» Publ. House, 2007. 373 p. 
12. Nan Jiang, Ran Shang, Heijman S.G.J., Luuk C. High-silica zeolites for adsorption of organic micro-pollutants in water treatment. A review Water Research, 2018, vol. 144, pp. 145-161.

13. Sulaiman K.O., Sajid M., Alhooshani K. Application of porous membrane bag enclosed alkaline treated Y-Zeolite for removal of heavy metal ions from water. Microchemical Journal, 2020, vol. 152, pp. 124-141.

14. Rassoulinejad-Mousavi S., Azamat J., Khataee A., Zhang Y Molecular dynamics simulation of water purification using zeolite MFI nanosheets. Separation and Purification Technology, 2020, vol. 234, 116080 .

15. Belova T.P. Adsorption of heavy metalions $\left(\mathrm{Cu}^{2+}, \mathrm{Ni}^{2+}, \mathrm{Co}^{2+}\right.$ and $\mathrm{Fe}^{2+}$ ) from aqueous solutions by natural zeolite. Heliyon, 2019, vol. 5,02320

16. Silva A.O.S., Souza M.J.B., Pedrosa A.M.G., Coriolano A.C.F., Araujo A.S. Development of HZSM-12 zeolite for catalytic degradation of high-densitypolyethylene. Microporous and Mesoporous Materials, 2017, vol. 244, pp. 1-6.

17. Kassargy C., Awad S., Burnens G., Kahine K. Experimental study of catalytic pyrolysis ofpolyethyleneand polypropylene over USY zeolite and separation to gasoline and diesel-like fuels. Journal of Analytical and Applied Pyrolysis, 2017, vol. 127, pp. 31-37.

18. Jambers Scandelai A.P., Zotesso J.P., Jegatheesan V., CardozoFilho L., Tavares C. Intensification of supercritical water oxidation $(\mathrm{ScWO})$ process for landfill leachate treatment through ion exchange with zeolite. Waste Management, 2020, vol. 101, pp. 259-267.

19. Abatal M., Córdova Quiroz A.V., Olguín M.T., VázquezOlmos A.R., Vargas J., Anguebes-Franseschi F., GiácomanVallejos $\mathrm{G}$. Sorption of $\mathrm{Pb}$ (II) from aqueous solutions by acidmodified clinoptilolite-rich tuffs with different $\mathrm{Si} / \mathrm{Al}$ ratios. Applied sciences, 2019, vol. 9, pp. 2415-2432

20. Manzoori J.L., Amjadi M., Abulhassan J. Ultra-trace determination of lead in water and food samples by using ionic liquid-based single drop microextraction - electrothermal atomic absorption spectrometry. Analytica Chimica, 2009, Acta 644, pp. $48-52$.

21. Portugal L.A., Ferreira H.S., Dos Santos W.N.L., Ferreira S.L.C. Simultaneous pre-concentration procedure for the determination of cadmium and lead in drinking water employing sequential multi- element flame atomic absorption spectrometry. Microchem, 2007, vol. 87 , pp. $77-80$.

22. Biasino J., Domínguez J.R., Alvarado J. Hydrogen peroxide in basic media for whole blood sample dissolution for determination of its lead content by electrothermal atomization atomic absorption spectrometry. Talanta, 2007, vol. 73 (5), pp. 962-964.

23. Sulistyaningrum I., Gitutami M.P., Istiningrum R.B., Siregar I.M. Comparison between the calibration and the standard addition methods in determining dissolved lead in Borobudurs control tanks water by Flame Atomic Absorption Spectrophotometry (F-AAS). Procedia Chemistry, 2015, vol. 17, pp. 70-74.

24. Zhou Q., Zhao N., Xie G. Determination of lead in environmental waters with dispersive liquid-liquid microextraction prior to atomic fluorescence spectrometry. Journal of Hazardous Materials, 2011, vol. 89 (1-2), pp. 48-53.

25. Yao-Min Liu, Feng-Ping Zhang, Bao-Yu Jiao, Jin-Yu Rao, GengLeng. Automated dispersive liquid-liquid microextraction coupled to high performance liquid chromatography - cold vapour atomic fluorescence spectroscopy for the determination of mercury species in natural water samples. Journal of Chromatography A, 2017, vol. 1493, pp. 1-9.

26. Radhakrishnan K., Panneerselvam P., Sivanesan S. Turn-on fluorescence sensor based detection of heavy metal ion using carbon dots graphitic-carbon nitride nanocomposite probe. Journal of Photochemistry and Photobiology A: Chemistry 389, 2019, $112204 \mathrm{~K}$.

27. Talio M.C., Alesso M., Acosta M., Acosta M.G., Fernández L.P. Sequential determination of lead and cobalt in tap water and foods samples by fluorescence. Talanta, 2014, vol. 127, pp. 244-249

28. Tarighat M. Orthogonal projection approach and continuous wavelet transform-feed forward neural networks for simultaneous spectrophotometric determination of some heavy metals in diet samples. Food Chemistry, 2016, vol. 192, pp. 548-556.

29. GOST 20255.1-89. Ionity. Metod opredeleniy astaticheskoy obmennoy emkosti [State Standart 20255.1-89. Ionites. Method for determining the static exchange capacity]. Moscow, Standartinform Publ., 2002. 5 p.

30. Rosenstein C., Hirsch S. Chemical analysis of plating solutions. Metal Finishing, 2002, vol. 100, pp. 509-554.

Received: 18 November 2020.

Information about the authors

Elmira A. Kambarova, doctoral student, Taraz State University named after M.Kh. Dualty.

Mikhail A. Gavrilenko, Dr. Sc., professor, National Research Tomsk Polytechnic University.

Nesipkhan A. Bektenov, Dr. Sc., professor, Kazakh National Pedagogical University named after Abay. 